\title{
Leonardo da Vinci y la nutrición humana
}

\author{
Ma Begoña Carretero Gómez \\ IES Valle de Lecrin (Dúrcal) Granada. España \\ E.mail:begocarretero@,botmail.com
}

[Recibido en Noviembre de 2009, aceptado en Julio de 2010]

\begin{abstract}
En esta experiencia trabajamos el tema la nutrición humana con una perspectiva diferente. Para ello hemos utilizado el libro de cocina "Notas de cocina de Leonardo da Vinci" de Shelagh and Routh, como texto base del que hemos extraído diferentes fragmentos, recetas y variada información de cómo era la forma de alimentarse y nutrirse en el Renacimiento. Se han realizado comparaciones entre la época actual y la de Leonardo. Para redondear la experiencia hemos cocinado algunas recetas extraídas del libro.
\end{abstract}

Palabras clave: nutrición; Leonardo da Vinci; cocina; dieta equilibrada; trastornos alimentarios.

\section{Leonardo da Vinci and the human nutrition}

We have done some activities related to human nutrition which have worked in a different way. We have used the recipes book “Leonardo's kitchen note books. Leonardo da Vinci's notes on cookery and table etiquette" by Shelagh and Routh, to obtain different fragments, recipes and information about Renascence, nutrition and alimentation. The students have made comparisons between current and Leonardo's nutrition. At the end we have cooked some of the Leonardo's recipes.

Keywords: nutrition; Leonardo da Vinci; cookery; healthy diet; bad nutrition habits.

\section{Introducción}

Una de las preocupaciones que hoy en día tiene el hombre es el conocer y poner en práctica una forma sana y equilibrada de alimentarse y por lo tanto de nutrirse. Leonardo da Vinci ya mostraba en algunos de sus escritos un gran interés por la nutrición, en ellos criticaba abiertamente las costumbres de la época y proponía lo que él consideraba una dieta adecuada. Teniendo en cuenta este auge y los problemas que actualmente se derivan de una mala conducta alimentaria, nos parece adecuado acercar al alumno, de una forma lo más experimental posible, a este tipo de conocimientos. De manera que aprendan ciencia de una forma atractiva, a la vez que conseguimos que su aprendizaje sea significativo.

\section{Justificación}

En la actualidad vemos como nuestra sociedad es constantemente bombardeada con ideas relacionadas con temas relativos a la nutrición, dieta equilibrada, peso ideal, alimentos enriquecidos, etc. Nuestros adolescentes están siendo fácilmente influidos por estas ideas, que en muchas ocasiones se encuentran lejos de la realidad y que les pueden llevar a seguir modelos de conducta erróneos pudiendo desembocar en trastornos alimenticios. Se hace imprescindible que desde

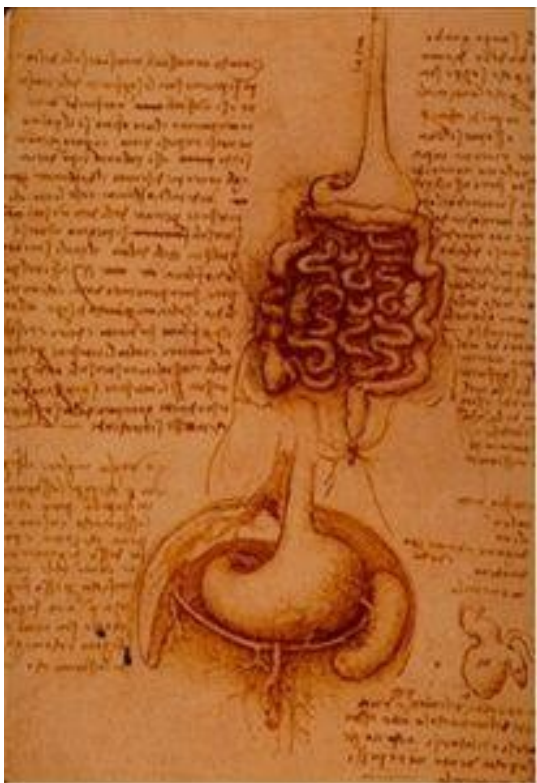

Figura 1. lámina de Leonardo da Vinci, aparato digestivo. 
asignaturas como la nuestra, colaboremos para que formación y alfabetización científica sean las adecuadas de manera que sean capaces de enfrentarse a estas situaciones con una actitud madura y responsable.

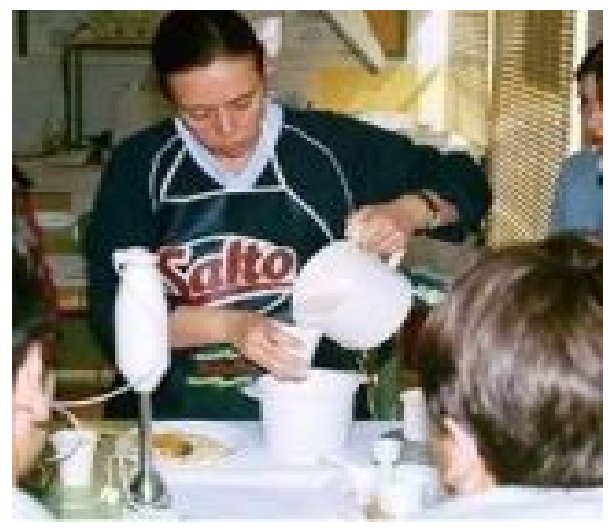

Figura 2. Cocinando pudín de plátano y miel.

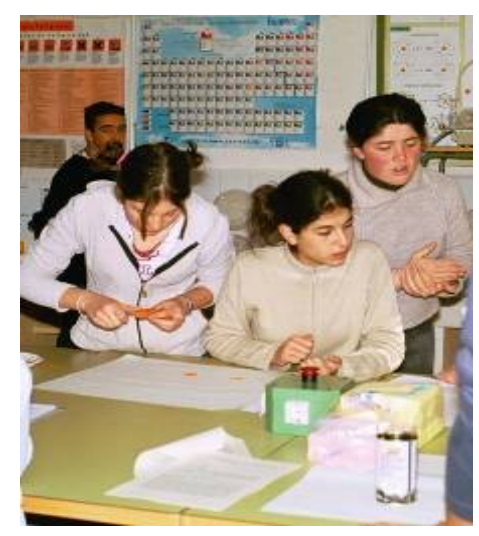

Figura 3. Alumnos preparando los ingredientes.

Por otra parte, hoy en día es bastante habitual que el profesorado tenga que trabajar con un alumnado que carece de interés por la asignatura y al que es difícil motivar. Esta es una de las mayores preocupaciones de la actividad del docente y son numerosas las alusiones a este problema, valgan como ejemplo García Borrás, (2005, 2010), Oliva, Matos, Bueno, Bonat, Dominguez, Vázquez, y Acevedo, (2004). Como bien dice Reid y Hodson, (1997), "El principal desafio de un profesor de ciencias es el motivar al alumno". Puesto que compartimos plenamente estas ideas, no es la primera vez que en el IES Isabel la Católica hemos desarrollado actividades en las que hemos tenido como intención prioritaria que el alumno participe de forma activa, manteniendo una actividad mental que le permita reorganizar, y modificar sus esquemas y conocimientos anteriores (Carretero 2006, Carretero 2007, Carretero y Sánchez 2008). Tanto en estas ocasiones, como en la experiencia que aquí describimos, hemos procurado que el alumno sea protagonista activo del proceso de enseñanza aprendizaje, es decir que no sea un mero receptor de ideas y conceptos. Creemos que las características de nuestro alumnado (secundaria obligatoria, centro de zona deprimida, alumnado con pocas expectativas e interés...) lo hacen un lugar adecuado para ponerlas en práctica.

El proceso de enseñanza-aprendizaje debe ser un proceso singular y original a la vez que participativo, (Aragón, 2004; Vázquez, 2004; Gómez e Insausti, 2005; Mateo, 2005; Gisasola, 1999). Las experiencias muestran como el alumnado se implica más, disfruta más, y aprende más con actividades participativas e investigativas, que cuando nos limitamos a desarrollar una clase meramente expositiva (Oliva, 2003; Gómez e Insausti, 2004;García Borrás 2006). Para ello hemos tomado como punto de partida actividades propias de nuestra vida cotidiana (elección de menús, cocinar, leer...), de esta forma hemos procurado acercarnos más a los alumnos e intentado hacer más llamativos los conceptos a trabajar. Así nos ha sido posible relacionar conocimientos, experiencias previas, intereses y necesidades del alumnado

Es necesario hacer que la ciencia sea funcional, es decir, que el alumnado sea consciente de la utilidad que tiene aquello que aprende. De la misma manera la ciencia debe ser atractiva y cumplir con sus intereses y expectativas, pero sin olvidar que a la vez debe ser ilustrativa. Debemos evitar que nuestras clases se conviertan en una "clase de cocina o de magia" en las que solamente nos limitamos a poner en práctica una receta determinada y en las que el alumnado no es consciente de cuales son nuestras pretensiones. Debemos promover el uso de la ciencia como una enseñanza en la que no solo busquemos a los posibles científicos del 
mañana, sino con la que colaboremos en la alfabetización científica de nuestros adolescentes para que así aprendan a ser críticos con toda la información que les llega del medio que les rodea, la ciencia forma parte del bagaje cultural del individuo y como tal hay que tratarla (Martín, 2002; García, 2005; Blanco, 2004; Varela y Martínez, 2005). Es fundamental el no perder la idea de que el alumnado debe ser responsable de su propio aprendizaje (Ausbel 1978, Santoveña 2004), teniendo el profesor el papel de guía y orientador en la construcción del pensamiento (Bruner, 1972; Coll, 1990)

De la misma manera hemos procurado que todo el trabajo se realice de una forma lo más interdisciplinar posible, desarrollando actividades relacionadas con otras áreas del conocimiento. La ciencia bien entendida, y como claramente podemos ver en el legado que nos ha dejado Leonardo da Vinci, es un compendio de saberes de la más variada naturaleza.

\section{Objetivos que perseguimos}

Como ya es de todos conocido, uno de los más destacados personajes del Renacimiento ha sido Leonardo da Vinci, caracterizado por su personalidad y genialidad. Son numerosas y muy variadas las áreas de conocimiento en las que trabajó, por lo que nos ha parecido un personaje idóneo para tomar como base a la hora de plantear esta experiencia. Para ello hemos tomado como punto de partida el libro "Notas de cocina de Leonardo da Vinci” dé Shelagh y Routh, donde son numerosas las menciones existentes sobre el interés que mostraba Leonardo a cerca de la nutrición y de la cocina. En este libro es posible leer fragmentos atribuidos a Leonardo en los que muestra una gran preocupación por el tipo de alimentación propia de la época, con comidas muy abundantes en las que predominaban los alimentos de origen animal (sobre todo carnes rojas) y en las que se tomaba poca verdura. Se sentía muy atraído por realizar una presentación atractiva de sus platos, además le gustaba experimentar con nuevas recetas. Son también destacables los extractos en los que hace mención a diferentes métodos de conservación así como al uso medicinal, culinario $y$ depurativo de algunas plantas. Todo esto nos ha movido a intentar despertar el interés de nuestro alumnado en el tema de la nutrición humana desde un punto de vista diferente, uniendo historia y ciencia de la mano de Leonardo da Vinci. aprovechando las ideas que Leonardo tiene acerca del tema para afianzar, corregir y ampliar los conocimientos que tienen sobre su propia nutrición.

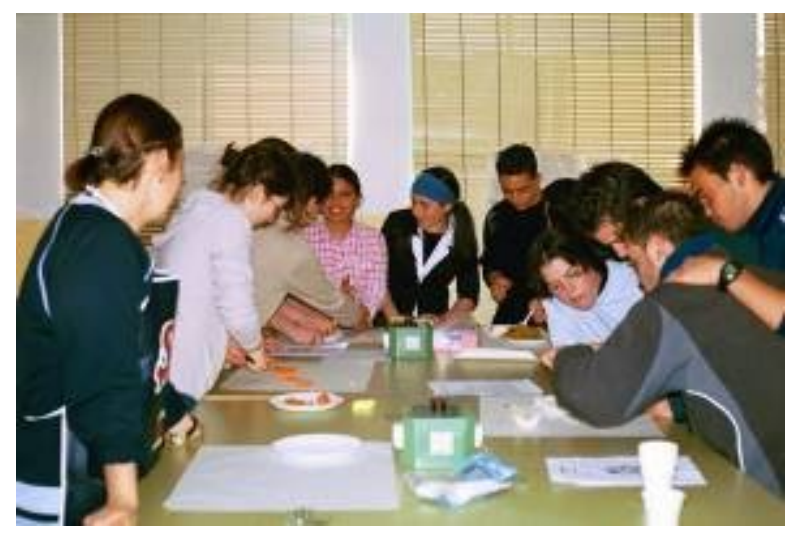

Figura 4. Alumnos en el laboratorio cocinando.

Las actividades programadas para realizar con el alumnado del IES Isabel la Católica de Guadahortuna, han ido encaminadas a que conocieran cual es la composición de los alimentos, las funciones básicas de sus biomoléculas constituyentes, como se realizan y cuales son los distintos procesos implicados en la nutrición humana, conocer los principios básicos de una dieta equilibrada etc. todo ello contando con la ayuda inestimable de Leonardo. Con estas actividades hemos perseguido que, además de la consecución de los objetivos que habitualmente interesan en relación con el tema de la nutrición, nuestros chicos y chicas fueran capaces de:

1. Descubrir la relación existente entre la ciencia y la historia. 
2. Descubrir una faceta desconocida de Leonardo da Vinci como es la de cocinero y dietista.

3. Reconocer los cambios sufridos en cuanto a la nutrición a lo largo de la historia comparando las diferentes formas de alimentación actual y renacentista.

4. Desarrollo de los conocimientos e ideas de Leonardo a cerca de la nutrición describiendo los banquetes y comidas renacentistas así como algunas de las recetas propuestas por él.

5. Reconocer los avances sufridos en cuanto a las mejoras en los métodos de cocinar y conservar los alimentos desde Leonardo hasta nosotros.

6. Aprender a elaborar dietas equilibradas

7. Desarrollar un espíritu crítico que les permita actuar de forma responsable llevando una nutrición adecuada.

8. Adquirir manejo y una mayor destreza en tareas culinarias.

9. Familiarizarse con diferentes procedimientos matemáticos (proporciones, cambios de unidades, cálculos numéricos).

\section{Actividades realizadas}

Hemos partido de la premisa de que una buena nutrición ayuda a mantener una buena salud, esta idea se ve claramente reflejada en los escritos atribuidos a Leonardo, en los que critica abiertamente las costumbres propias de la época. Tomando como punto de partida este tipo de comentarios, hemos desarrollado actividades de diversa índole (investigativo, manipulador, divulgativo...) que tenían en común el utilizar la historia de la ciencia, y en concreto la figura de Leonardo da Vinci para ampliar los conocimientos y habilidades científicas de nuestro alumnado.

Para iniciarnos en el tema y poder "ubicarnos" tanto alumnado como profesora, se comenzó realizando un cuestionario inicial y puesta en común para poner de manifiesto cuales eran los conocimientos previos que tenían sobre la nutrición. Es importante comprobar que diferencian correctamente los conceptos de nutrición y alimentación, tan habitualmente utilizados como sinónimos sin serlo. Nos ha interesado saber que piensan ellos sobre las necesidades básicas de su organismo, y sobre todo nos ha parecido fundamental detectar posibles casos en los que la nutrición no es la adecuada. De la misma manera este tipo de cuestionario nos puede ayudar modificar muchos de los conceptos erróneos, tabúes y dudas que los alumnos puedan tener.

Posteriormente se realizó una lectura comentada de diferentes fragmentos del libro "Notas de cocina de Leonardo da Vinci". Previamente la profesora ha seleccionado aquellos fragmentos que podrían ser de mayor interés y se los presenta a sus alumnos. Estos fragmentos destacan por ser curiosos, otros por ser simpáticos, por tener errores conceptuales, por ser interesantes desde el punto de vista nutricional....Se han leído en clase y se ha realizado una puesta en común en la que el alumnado ha expresado sus ideas en relación a los mismos. Discutiendo sobre si las ideas planteadas por Leonardo son o no adecuadas y argumentando sus opiniones. De la misma manera se han utilizado estos textos para que el alumnado extraiga información.

Para profundizar en la figura de Leonardo, nuestros chicos y chicas, utilizando diversas fuentes de información, han elaborado una biografía sobre este personaje. La profesora les ha propuesto investigar la figura de Leonardo como personaje del Renacimiento de manera que ellos mismos obtengan la información necesaria para escribir como trabajo propio una biografía. A partir de los datos que ellos han ido encontrando y aportando, se ha hecho un 
compendio de los mismos y se ha escrito una biografía general que es la que acompaña el cuaderno del alumno.

Como es lógico a lo largo de la actividad se manejan términos que hasta ahora han sido desconocidos para ellos, de manera que de forma paralela el alumnado ha dedicado un poco de tiempo a la elaboración de un pequeño diccionario con dichos términos. A lo largo de las lecturas tanto de los fragmentos del libro, como de las recetas, o incluso de las pequeñas nociones de teoría que les han sido dadas, el alumnado ha ido registrando su propio glosario de terminología tecno-científica.

Por otra parte se ha realizado la búsqueda de información relacionada con el tipo de banquetes propios del Renacimiento. Teníamos gran interés en que obtuvieran información en cuanto al número de platos, tipos de comidas, duración de los mismos, costumbres en la mesa. Leonardo era enormemente crítico con la sociedad de su época, de manera que es una buena oportunidad para que el alumnado aumente su capacidad crítica, a la vez que se hace consciente de las características propias de una buena y equilibrada nutrición.

Otro punto interesante ha sido la recopilación de recetas de cocina atribuidas a Leonardo. Además de las que están incluidas en el libro "Notas de cocina de Leonardo da Vinci" se han buscado platos propios de la época. De todos ellos se han estudiado los ingredientes y las proporciones de los mismos. De los ingredientes desconocidos se han realizado las correspondientes investigaciones para descubrir con cuál de los alimentos propios de la actualidad se corresponden.

Nos pareció interesante que nuestro alumnado aprendiese a manejar tablas de alimentos, a diseñar dietas y a reconocer una dieta adecuada de la que no lo es, así que les propusimos un estudio nutricional del menú elaborado para la sobrina del Señor Sforza, para quien trabajaba Leonardo. Como nuestros chicos y chicas pudieron leer en los fragmentos del libro que les proporcionamos, hay dos menús. Uno es el que el seños Sforza quiere que Leonardo cocine y otro es el que propone el propio Leonardo. Se tomaron como base ambas propuestas y se realizó un estudio nutricional comparativo. Posteriormente se les sugirió que elaborasen un menú alternativo intentando mantener los principios nutricionales básicos. Acudimos a que el alumno usara su propia imaginación y a partir de sus propias vivencias elaboraran una propuesta de menú para una boda. Esta actividad ha sido muy útil para que comprueben que, aunque ellos estaban convencidos de que lo normal es llevar una nutrición adecuada, es muy fácil salirse de la norma.

Ya hemos mencionado anteriormente que Leonardo estaba muy interesado en la utilidad de algunas especies vegetales así que se procedió a la búsqueda de información a cerca de las propiedades que Leonardo atribuye a algunos productos. Hoy en día algunos de estos remedios caseros son conocidos y se usan habitualmente, otros no lo son tanto. Puesto que nuestro centro escolar se ubica en una zona eminentemente rural, es muy común que la tradición de padres a hijos haya transmitido este tipo de saberes populares. El alumnado ha recopilado el uso (medicinal, especias, insecticida, etc.) que se le da a diversas plantas. En algunos casos se ha podido comprobar la coincidencia entre lo indicado por Leonardo y lo que actualmente hacemos.

Ya en la fase final de la experiencia se procedió a la búsqueda de recetas actuales que se corresponden con las atribuidas a Leonardo. En muchas de las recetas de Leonardo los ingredientes no estaban a nuestro alcance, algunos incluso eran desconocidos para nosotros, de manera que realizamos una selección de recetas que tenían algún parecido o que coincidían prácticamente en su totalidad con platos que son habituales en nuestras mesas. 
Como colofón y a manera de celebración preparamos algunas de estas recetas. Una vez seleccionados los platos más asequibles en todos los sentidos (facilidad, pocos ingredientes,...) Nos decidimos a cocinar algunos de ellos. Lo mismo que Leonardo estaba muy preocupado por la presentación adecuada de sus platos, hemos hecho mucho hincapié en que no solo fueran agradables en su sabor, sino también en su aspecto. Por ello han tenido que esforzarse en su decoración.

La actividad final consistió en la realización de descripciones del trabajo realizado. Hemos vuelto a hacer una puesta en común, donde se han realizado diversas presentaciones tanto de los conocimientos adquiridos, de los materiales elaborados, así como de las recetas cocinadas.

\begin{tabular}{|l|}
\hline \multicolumn{1}{|c|}{ RELACIÓN DE ACTIVIDADES REALIZADAS } \\
\hline Cuestionario inicial para comprobar conocimientos previos \\
\hline Búsqueda de información sobre la vida y obra de Leonardo da Vinci \\
\hline Elaboración de una biografía del personaje \\
\hline Elaboración de un diccionario con nuevos términos \\
\hline Lectura comentada de diversos fragmentos del libro "Notas de cocina de Leonardo da Vinci" \\
\hline Puesta en común en relación a los textos leídos \\
\hline $\begin{array}{l}\text { Búsqueda de información relativa a los banquetes típicos del Renacimiento y platos propios de la } \\
\text { época }\end{array}$ \\
\hline Recopilación de recetas atribuidas a Leonardo \\
\hline Uso e interpretación de tablas nutricionales \\
\hline Estudio nutricional de varios menús \\
\hline Elaboración de un menú propio que sea adecuado a las necesidades de cada uno \\
\hline Estudio del uso medicinal que de algunas plantas \\
\hline Elaboración de algunas de las recetas recopiladas. \\
\hline
\end{tabular}

Además de los recursos personales que tanto el profesor como el alumnado pudieron aportar durante el desarrollo de la actividad, usamos distinto tipo de recursos materiales:

- Periódicos, revistas, así como distinta información bibliográfica a cerca de los conocimientos sobre nutrición y dietas elaboradas por Leonardo da Vinci. Fragmentos de distintas biografías sobre Leonardo en las que nos acercamos a los conocimientos científicos que este poseía.

- Etiquetas y folletos publicitarios de diferentes alimentos.

- Material de cocina necesario para la realización del "taller de cocina".

- Tablas nutricionales de alimentos.

- Enciclopedias y otros libros de consulta.

- Internet.

- Audiovisuales.

La mayoría del tiempo se ha realizado la actividad en el aula propia del grupo o clase, salvo en aquellos casos en los que los alumnos debían hacer consultas bien en la biblioteca, bien en la sala 
de informática. Por su parte las recetas de cocina se elaboraron en el laboratorio de ciencias naturales.!

Esta experiencia ha sido muy enriquecedora y, entre otros, nos ha permitido tratar los siguientes temas transversales:

- Educación para la salud: una dieta sana y equilibrada para mantener nuestro cuerpo en unas condiciones óptimas. Hemos podido tratar problemas o alteraciones alimentarias como es el caso de la anorexia, la malnutrición, la bulimia etc. El funcionamiento correcto de los órganos relacionados con la nutrición implica un funcionamiento correcto del organismo. Los órganos de los sentidos (vista, olfato, gusto) están íntimamente ligados al proceso de alimentación un buen mantenimiento e higiene de los mismos producirá mayor satisfacción en dicho proceso.

- Educación para la convivencia: los alumnos han aprendido a respetar distintas formas de alimentación que en muchas ocasiones van relacionadas con determinadas convicciones religiosas. En otras ocasiones los hábitos alimenticios vienen dados por formas de pensar y costumbres. Debemos ser tolerantes ante la gran variedad de comportamientos existentes.

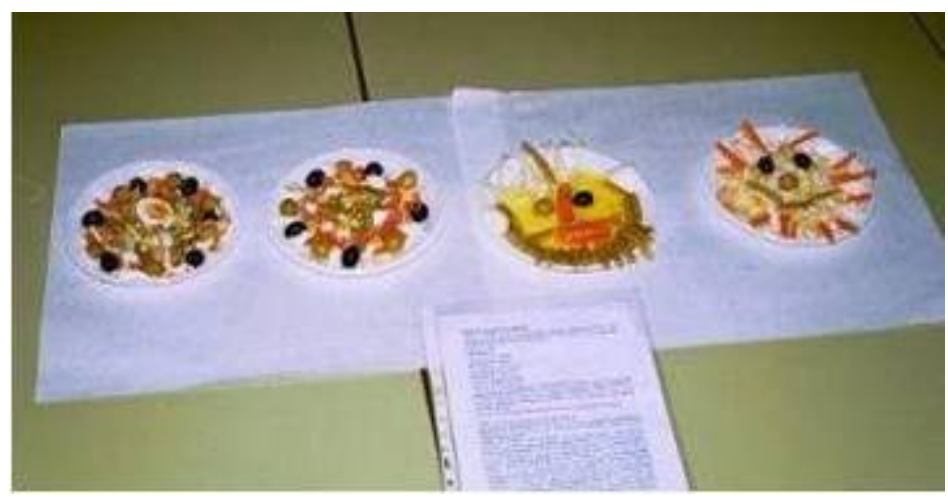

Figura 5. Diferentes platos de crudos.

- Educación no sexista: las diferencias fisiológicas y anatómicas entre hombre y mujer determinan que las necesidades energéticas y nutritivas de ambos sean distintas, el alumno debe de comprender que no por ello el hombre y la mujer son individuos diferentes. La fisiología y la anatomía entre hombre y mujer es común, no existen por tanto diferencias anatómicas entre ambos sexos. El reparto de tareas en el hogar y en este caso concreto las relacionadas con la alimentación, y preparación de los alimentos.

\section{Conclusiones}

Como profesores que somos sabemos que llevar a cabo actividades de esta naturaleza suponen al docente un esfuerzo extra en su trabajo diario. Esto hace que cuando las planteamos nos sintamos un poco reacios a llevarlas a cabo. Si embargo se hacen necesarias si partimos de la idea de que si el alumnado participa de una forma activa, dinámica y motivada, el proceso de enseñanza aprendizaje es más asequible para ellos, ya que de esta forma lo hacemos más cercano a su actividad diaria (García 2005, Bendala y Pérez, 2004; Carretero, 2006).

Son muchas más las ventajas que los inconvenientes que podemos obtener de ellas. Se convierten en propuestas agradables y amenas en las que el alumnado se encuentra encantado de participar puesto que se adaptan mejor a sus necesidades. Sin que ellos se den cuenta, se establece una relación de cooperación entre alumnos de manera que la base del trabajo es la ayuda mutua. De esta forma aquellos que presentan mayores dificultades superan con más 
facilidad los posibles obstáculos. Los chicos y chicas han trabajado en grupo de una forma coordinada, ayudándose mutuamente y desarrollando así un espíritu de colaboración. Han trabajado con gran interés y esfuerzo, siempre en la medida de sus posibilidades. Al igual que en experiencias anteriores (Carretero, 2006; Carretero y Sánchez, 2008) hemos constatado que académicamente el alumno que lleva buena trayectoria académica la mantiene mientras que los menos aventajados, y salvo las excepciones, han mejorado mucho sus resultados al beneficiarse de la ayuda recibida por parte de sus compañeros (García e Insausti, 2005).

Ha habido un diálogo constante entre profesorado y alumnado de manera que esta comunicación ha sido fluida, intercambiando gran cantidad de ideas donde el aprendizaje ha sido mutuo. Esta comunicación ha sido fundamental para el desarrollo del proceso de enseñanza-aprendizaje y ha traído consigo el alto grado se satisfacción mostrado por todos, estableciéndose una relación entre alumnado y profesor que en otras condiciones habría sido más difícil. Con esta experiencia el alumnado ha podido:

- Comprobar como han ido cambiando los diferentes hábitos sociales relacionados con la alimentación mediante la lectura de diferentes textos atribuidos a Leonardo y extraídos del libro "Notas de cocina de Leonardo da Vinci". En este libro se hace una fuerte crítica a las costumbres alimenticias de la época, lo cual hemos aprovechado para despertar en ellos el

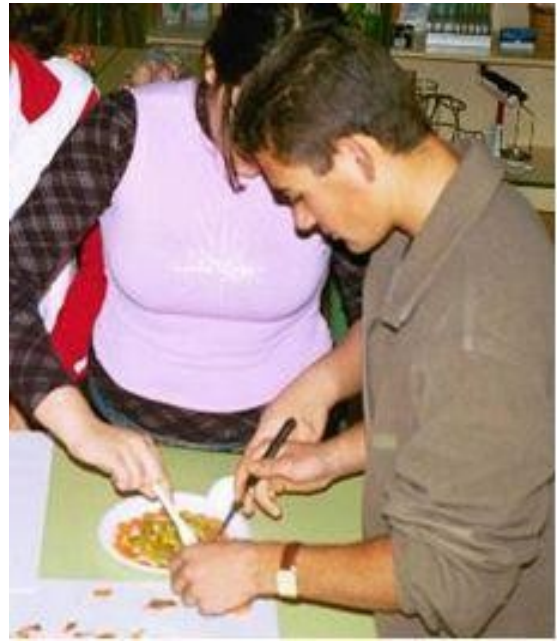

Figura 6. Alumnos decorando el plato. interés por seguir una dieta sana y equilibrada.

- Realizar estudios comparativos entre las recetas sugeridas por Leonardo, y las que actualmente seguimos a la hora de elaborar platos semejantes.

- Calcular el valor y aporte nutritivo de las recetas trabajadas. De esta manera han aprendido a realizar cálculos nutricionales y energéticos de los diferentes platos, comprobando si se atienen o no a las recomendaciones generales sobre una nutrición adecuada.

- Elaborar dietas equilibradas respetando la proporción de nutrientes. Simultáneamente han aprendido a ser críticos con la información recibida, de manera que han sido conscientes de la evolución y cambios en la mejora de las costumbres culinarias de la sociedad desde el Renacimiento hasta nuestros días.

- Familiarizarse con el uso de vocabulario científico, a la vez que han sido capaces de diferenciar entre una descripción científica y otra literaria, aprendiendo algunos tecnicismos. Han manejado diferentes tipos de textos escritos, así como han utilizado diferentes fuentes de información (enciclopedias, libros de texto, Internet, tablas etc.) para buscar los datos necesarios.

$\mathrm{Al}$ igual que Leonardo fue un hombre polifacético, hemos intentado que nuestra experiencia sea lo más interdisciplinar posible relacionando saberes diferentes entre sí. Se ha salido del encasillamiento que suponen las diversas asignaturas, para tratar un mismo tema de una manera más interdisciplinar ya que la experiencia abarca conocimientos propios de otras áreas (matemáticas, lengua, ciencias sociales, etc.). Así por ejemplo podemos destacar su relación con: 
a) Ciencias Sociales e Historia: estudio de las costumbres de la época, los tipos de cultivos de la zona, ganadería y mercados. Estudio del Renacimiento como periodo de florecimiento en multitud de disciplinas. Descubrimiento de Leonardo da Vinci como un personaje polifacético.

b) Educación Física: se tomó el eje de una buena nutrición como base para un buen funcionamiento del organismo, relacionando la actividad física con el gasto energético y con los nutrientes aportados por los alimentos.

c) Lengua castellana y literatura: se analizan los textos correspondientes a las distintas recetas de cocina propuestas por Leonardo y otras propias de la época. Lectura de fragmentos del libro "Notas de cocina de Leonardo da Vinci".

d) Matemáticas: estudio de proporciones y unidades de medida.

e) Educación Plástica y visual: elaborando y decorando platos para hacerlos agradables a la vista.

\section{Referenfias bibliográficas}

ARAGÓN, MM; BONAT, M.; CERVERA, J; MATEO, J.; OLIVA, JM, (1998). Las analogías como estrategia didactica en la en señanza de la Física y de la Química. En E. Banet y A. de Pro (coods), Investigación e innovación en la enseñanza de las ciencias. La pobla de Segur: DM

ARAGÓN, M.M. (2004). La ciencia de lo cotidiano. Revista Eureka sobre la Enseñanza y Divulgación de las Ciencias, 1(2), 109-121. En línea en: http://reuredc.uca.es.

AUSUBEL, D. (1978) Psicología educativa. Un punto de vista cognitivo. México; Trillas

BENDALA MUÑOZ, M. Y PÉREZ ORTEGA J.A. (2004) Educción ambiental: praxis científica y vida cotidiana. Descripción de un proyecto. Revista Eureka sobre la Enseñanza y Divulgación de las Ciencias, 1(3), 233-239. En línea en: http://reuredc.uca.es/.

BLANCO, A. (2004). Relaciones entre la educación científica y la divulgación de la ciencia. Revista Eureka sobre la Enseñanza y Divulgación de las Ciencias, 1(2), 70-86. En línea en: http://reuredc.uca.es.

BRUNER, J. (1972) Hacia una teoría de la instrucción. México: Uteha.

CARRETERO, M.B. (2006). Celebremos el primer centenario de la teoría de la relatividad conociendo a los científicos y su trabajo. Revista Eureka sobre enseñanza y Divulgación de las Ciencias, 3(2), 287-299. En línea en: http://reuredc.uca.es.

CARRETERO, M.B. (2007). Wolfgang Amadeus Mozart nos ayuda ha estudiar anatomía. Revista Eureka sobre Enseñanza y Divulgación de las ciencias 4(1), 176-188. En línea en: http://reuredc.uca.es.

CARRETERO, M.B. Y SANCHEZ M.A. (2008). Talleres para celebrar la semana de la ciencia. Revista Eureka sobre enseñanza y Divulgación de las Ciencias, 5(1), pp. 61-73. http://reuredc.uca.es

COLL, C. (1990) Aprendizaje escolar y construcción del conocimiento. Barcelona: Paidós.

GARCÍA BORRÁS, F. J. (2005) La serie CSI como metáfora de algunas facetas del trabajo científico. Revista Eureka sobre enseñanza y Divulgación de las Ciencias, 2(3), 374-387. En línea en: $\underline{\text { http://reuredc.uca.es }}$

GARCÍA BORRÁS, F. J. (2006). Cuando los mundos chocan. Revista Eureka sobre enseñanza y Divulgación de las Ciencias, 3(2), 268-286. En línea en: 


\section{http://reuredc.uca.es}

GARCÍA CARMONA, A. (2005). Relaciones CTS en el estudio de la contaminación atmosférica: una experiencia con estudiantes de secundaria. Revista Electrónica de enseñanza de las ciencias, 4(2), artículo 3. En línea en: http://www.saum.uvigo.es/reec.

GUISASOLA, J. (1999) Implicaciones de la investigación educativa en la enseñanzaaprendizaje de las ciencias experimentales. En: LAFUENTE JV, RUIZ DE GAUNA P, SOLANO D (eds.). Enseñanza y Medicina. Mosaico de de reflexiones en torno a la docencia de las ciencias médicas. Leioa (Bizkaia): Euskal Herriko Unibertsitateko Argitalpen Zerbitzua, 51-62.

GÓMEZ GARCÍA, J.A. e INSAUSTI TUÑÓN, M.J. (2004). El ciclo reflexivo cooperativo: un modelo didáctico para la enseñanza de las ciencias. Revista electrónica de enseñanza de las ciencias, 3(2), artículo 2 .

GÓMEZ GARCÍA, J.A. e INSAUSTI TUÑÓN, M.J. (2005). Un modelo para la enseñanza de las ciencias: análisis de datos y resultados. Revista Electrónica de Enseñanza de la Ciencias, 4(3), artículo 6.

MATEO SÁNCHEZ, J. (2005). La atención a la diversidad en ciencias a través de materiales curriculares adaptados. Revista Eureka sobre la Enseñanza y Divulgación de las Ciencias, 2(3), 416-429. En línea en: http://reuredc.uca.es.

MARTÍN DÍAZ, M.J. (2002). Enseñanza de las ciencias ¿para qué? Revista electrónica de la enseñanza, 1(2) artículo1. http://www.saum.uvigo.es/reec/.

OLIVA, J.M (2003) Rutinas y guiones del profesorado de ciencias ante el uso de analogías como recurso en el aula. Revista Electrónica de enseñanza de las ciencias. 2(1) artículo2 En línea en: http://www.saum.uvigo.es/reec

OLIVA, J.; MATOS, J.; BUENO, E.; BONAT, M.; DOMINGUEZ, J.; VÁZQUEZ, A. Y ACEVEDO, J.A. (2004). Las exposiciones científicas escolares y su contribución al ámbito afectivo de los alumnos participantes. Enseñanza de las ciencias, 22(3), 425440.

REID, D.J.; HODSON, D. (1997) Ciencia para todos en secundaria. Madrid: Narcea de Ediciones S.A.

ROUTH SHELAG AND JONATHAN ROUTH (1987).Notas de cocina de Leonardo da Vinci, la afición desconocida de un genio. Madrid: temas de hoy.

SANMILLÁN, C. 500 recetas fáciles. ed: Grafalco

SANTOVEÑA, S. Ma (2004). Metodología didáctica en entornos virtuales de aprendizaje. Etica@net.AñoII número 3:

En linea: www.ugr.es/sevimeco/revistaeticanet/numero3/Articulos/metodologia\%20didactica.pdf

VARELA NIETO, M.P. y MARTÍNEZ MONTALBÁN J.L. (2005). "Jugando" a divulgar la física con juguetes. Revista Eureka sobre la Enseñanza y Divulgación de las Ciencias, 2(2) 234-240. En línea en: http://reuredc.uca.es.

VÁZQUEZ GONZÁLEZ, C. (2004). Reflexiones y ejemplos de situaciones didácticas para una adecuada contextualización de los contenidos científicos en el proceso de enseñanza. Revista Eureka sobre la Enseñanza y Divulgación de las Ciencias, 1(3), 214223. En línea en: http://reuredc.uca.es. 


\section{ANEXO: CUADERNO DEL ALUMNO}

Este cuaderno esta estructurado en varias partes. En la primera, se presenta una breve biografía del personaje. En la segunda, se trata el tema de la nutrición y la dietética, comparando los conocimientos actuales y los de Leonardo. En la tercera, se proponen algunas de las recetas que Leonardo incluye en sus escritos o en su caso, aquellas parecidas y que se cocinan en la actualidad, para que sea nuestro alumnado el encargado de elaborarlas.

\section{Parte I: Biografía de Leonardo da Vinci}

\section{Parte II: Leonardo da Vinci y la nutrición}

A continuación el cuaderno del alumno presenta textos que se han extraído del libro "Notas de cocina de Leonardo da Vinci" (Shelagh and Routh, 1987). En ellos se hace mención a algunos manuscritos atribuidos a Leonardo y en los que se puede adivinar su preocupación por seguir una dieta sana y equilibrada. Estos fragmentos versan sobre los siguientes temas:

- De las ventajas de una dieta moderada.

- De los platos sencillos.

- De la conservación de los alimentos.

- De las cantidades más provechosas de alimentos y líquidos que han de tomarse cada día.

- De una selección equilibrada de alimentos.

- De las hierbas.

- Mis platos sencillos.

- Menú para la boda de la sobrina del señor Sforza.

\section{Cuestiones y actividades a realizar por nuestro alumnado:}

1- ¿Podrías adivinar tras leer este trabajo, de que tipo de dieta era partidario Leonardo?

2- ¿Qué método de conservación de alimentos propone usar Leonardo? Infórmate sobre el fundamento de dicho método.

3- ¿Coincide el porcentaje de alimentos a ingerir diariamente con los que hoy en día son aconsejables? ¿En que se diferencia?

4- ¿A qué grupo de alimentos se refiere Leonardo al hablar sobre los únicos tres alimentos con los que un hombre puede alimentarse sin sufrir déficits? ¿Compartes su idea? Explícalo.

5- ¿Cuál piensas que es la razón de que muera su amigo Benedetto Garvi que según el seguía la anterior dieta?

6- ¿Es adecuada la proporción de alimentos sólidos y líquidos que han de tomarse al día, de la que habla Leonardo, según las normas dietéticas hoy en día establecidas?

7- Diferencia entre los términos alimentación y nutrición, nutriente y alimento.

8- ¿Qué significado tiene la frase: "hay muchas formas de alimentarse pero solo una de nutrirse".

9- Haz un estudio detallado de los nutrientes (Kcal, azúcares, lípidos, proteínas) que aporta el menú diseñado por Leonardo para la boda de la sobrina del señor Ludovico Sforza. Debes 
usar las tablas que se adjuntan para tomar los datos necesarios. (Preséntalo según tabla inferior).

¿Están presentes todos los grupos de alimentos? En caso negativo, ¿cuál o cuales faltan?

10- Elabora tu propio menú para dicha boda cuidando que se cumplan las proporciones adecuadas.

11- Busca información sobre enfermedades relacionadas con la mala nutrición.

\begin{tabular}{|l|l|l|l|l|l|}
\hline Alimento & cantidad & Proteínas & lípidos & Azúcares & Kcal \\
\hline & & & & & \\
\hline Total & & & & & \\
\hline
\end{tabular}

\section{Parte III: taller de cocina, el renacimiento y Leonardo entre fogones}

Esta parte consta de una breve introducción en la que se describe cual era la estructura más típica de los banquetes medievales y renacentistas (número de platos, orden etc.) así como de una serie de recetas que han sido utilizadas para cocinar ellos mismos. Estas recetas de cocina han sido extraídas del libro de "notas de cocina de Leonardo da Vinci". Detrás de cada una de ellas describimos la receta actual que consideramos más parecida, algunas de ellas como ocurre con el plato de crudos o el mazapán son casi idénticas.

- Receta 1: pudín blanco (albóndigas).

- Receta 2: mazapán.

- Receta 3: huevos cocidos, (huevos rotos).

- Receta 4: plato de crudos (ensalada de judías verdes).

- Receta 5: pudín de plátano y miel.

- Receta 6: pollo con hinojo.

- Receta 7: omelette de naranjas para prostitutas y rufianes (natillas de naranja). 